\title{
Two-Dimensional Variational Analysis of Near-Surface Moisture from Simulated Radar Refractivity-Related Phase Change Observations
}

\author{
Ken-ichi SHIMOSE ${ }^{1,2}$, Ming XUE*1,3, Robert D. PALMER ${ }^{3,4}$ Jidong GAO $^{1,5}$, \\ Boon Leng CHEONG ${ }^{4}$, and David J. BODINE ${ }^{3,4}$ \\ ${ }^{1}$ Center for Analysis and Prediction of Storms, University of Oklahoma, Norman OK, USA \\ ${ }^{2}$ Department of Earth and Planetary Sciences, Kyushu University, Fukuoka, Japan \\ ${ }^{3}$ School of Meteorology, University of Oklahoma, Norman OK, USA \\ ${ }^{4}$ Atmospheric Radar Research Center, University of Oklahoma, Norman OK, USA \\ ${ }^{5}$ National Severe Storms Laboratory, NOAA, Norman OK, USA
}

(Received 28 April 2012; revised 29 June 2012)

\begin{abstract}
Because they are most sensitive to atmospheric moisture content, radar refractivity observations can provide high-resolution information about the highly variable low-level moisture field. In this study, simulated radar refractivity-related phase-change data were created using a radar simulator from realistic highresolution model simulation data for a dryline case. These data were analyzed using the 2DVAR system developed specifically for the phase-change data.

Two sets of experiments with the simulated observations were performed, one assuming a uniform target spacing of $250 \mathrm{~m}$ and one assuming nonuniform spacing between $250 \mathrm{~m}$ to $4 \mathrm{~km}$. Several sources of observation error were considered, and their impacts were examined. They included errors due to ground target position uncertainty, typical random errors associated with radar measurements, and gross error due to phase wrapping. Without any additional information, the 2DVAR system was incapable of dealing with phase-wrapped data directly. When there was no phase wrapping in the data, the 2DVAR produced excellent analyses, even in the presence of both position uncertainty and random radar measurement errors. When a separate pre-processing step was applied to unwrap the phase-wrapped data, quality moisture analyses were again obtained, although the analyses were smoother due to the reduced effective resolution of the observations by interpolation and smoothing involved in the unwrapping procedure. The unwrapping procedure was effective even when significant differences existed between the analyzed state and the state at a reference time. The results affirm the promise of using radar refractivity phase-change measurements for near-surface moisture analysis.
\end{abstract}

Key words: radar refractivity, variational analysis, low-level moisture

Citation: Shimose, K., M. Xue, R. D. Palmer, J. D. Gao, B. L. Cheong, and D. J. Bodine, 2013: Twodimensional variational analysis of near-surface moisture from simulated radar refractivity-related phase change observations. Adv. Atmos. Sci., 30(2), 291-305, doi: 10.1007/s00376-012-2087-7.

\section{Introduction}

The amount and distribution of moisture in the atmosphere are among the most important factors affecting the prediction of mesoscale and storm-scale weather (e.g., McPherson et al., 1997), particularly for quantitative precipitation forecasting (QPF; Emanuel et al., 1995; Fritsch et al., 1998; Droegemeier et al., 2000). High-resolution measurements of moisture within the boundary layer (BL) are even more impor-

\footnotetext{
*Corresponding author: Ming XUE, mxue@ou.edu

(C) China National Committee for International Association of Meteorology and Atmospheric Sciences (IAMAS), Institute of Atmospheric Physics (IAP) and Science Press and Springer-Verlag Berlin Heidelberg 2013
} 
tant because BL moisture is the essential fuel for convection and precipitation.

BL moisture has high spatial and temporal variability, resulting from often inhomogeneous lower boundary forcing and BL convective eddies and rolls (Weckwerth et al., 1996; Weckwerth and Parsons, 2006; Xue and Martin, 2006a). Typical surface observation networks are too coarse to resolve the fine-scale structures in BL moisture. Consequently, BL moisture is poorly characterized by existing observation platforms (Weckwerth et al., 2004).

Ground-based GPS receiver networks provide only path-integrated quantities and lack resolution in the BL. The GPS-slant-path water vapor measurements are most effective at the mid to upper levels where slant paths intersect (Liu and Xue, 2006). At the same time, the low-orbiting-satellite-based GPS occultation data generally do not reach the surface and have poor horizontal resolution (Ware et al., 1996; Anthes et al., 2008). Mesoscale surface observational networks (e.g., Brock et al., 1995) are currently the best platform for near-surface moisture observations, but their spatial resolution may not be sufficient to resolve fine-scale structures important for convective initiation (Weckwerth et al., 2004). Therefore, additional highresolution moisture measurements within the BL are needed to fill the data gap, and such data have the potential to significantly improve convective initiation and quantitative precipitation forecasting.

High-resolution near-surface water vapor measurements can be derived from radar-based refractivity measurements utilizing returns from fixed ground targets (Fabry et al., 1997; Weckwerth et al., 2005). Effective assimilation of radar refractivity measurements into a NWP model is a relatively new research area that requires much research. The actual impact of such observations on storm-scale NWP also needs investigation, which is most effectively accomplished by combining meteorological and engineering knowledge. Before testing data from a new platform, it is valuable to perform experiments using simulated data through observing system simulation experiments (OSSEs; Lord et al., 1997), where observations as well as error properties can be simulated and systematically evaluated. The OSSE framework also provides exact information about error properties from different sources.

In this study, we aim to develop the capability to analyze refractivity-derived measurements of nearsurface moisture based on a variational method. We first test such a capability using simulated refractivity and phase-change data. A previously modeled case from the 2002 International $\mathrm{H}_{2} \mathrm{O}$ Project (IHOP_2002) field experiment serves as the truth. Refractivity and phase-change data are simulated using a realistic radar refractivity emulator that includes simulation of observation errors from several possible sources. The data are analyzed using a specially designed twodimensional variational (2DVAR) system that properly accounts for observation and background errors. The phase-wrapping issue is investigated in some detail, including the effect of phase wrapping on the analysis quality. A practical phase-unwrapping procedure is applied to the simulated data. The unwrapped data are analyzed with a proper error estimate. The quality of the analysis is evaluated by comparing the analyzed moisture fields with the truth.

The rest of this paper is organized as follows: radar refractivity measurements and the generation of simulated radar phase-change difference observations and their associated error are discussed in section 2 . The 2DVAR analysis method is described in section 3 , and the results of analysis experiments are presented in section 4. Further discussion of our scheme's effectiveness is given in section 5 through sensitivity experiments. A summary is provided in section 6 .

\section{Radar refractivity and phase-change differ- ence measurements and observation simu- lation}

\subsection{Radar refractivity measurements}

In a vacuum, an electromagnetic (EM) wave travels at the speed of light $\left(c=3 \times 10^{8} \mathrm{~m} \mathrm{~s}^{-1}\right)$. In the atmosphere, however, the speed of the wave decreases by a factor equivalent to the index of refraction of the air, denoted by $n$, which is given by

$$
n=1+77.6 \times 10^{-6}\left(\frac{P}{T}+4810 \frac{P_{\mathrm{w}}}{T^{2}}\right),
$$

where $T$ is the temperature in degrees Kelvin, $P$ is the total pressure and $P_{\mathrm{w}}$ is the partial pressure of water vapor both in hPa. In Eq. (2.1), the values of $n$ are very close to 1 . Refractivity, $N$, is a more convenient quantity that is related to the refractive index as defined by Bean and Dutton (1968):

$$
N \equiv(n-1) \times 10^{6}=77.6\left(\frac{P}{T}+4810 \frac{P_{\mathrm{w}}}{T^{2}}\right) .
$$

At warmer temperatures, $N$ is most sensitive to variability in $P_{\mathrm{w}}$.

A technique was developed by Fabry et al. (1997) to make radar measurements of near-surface refractivity, thereby deducing near-surface moisture. This technique is briefly described here. The phase of the backscattered radar signal after coherent detection is related to the path-integrated refractive index by the 
following equation,

$$
\phi(r, t)=-\frac{4 \pi f}{c} \int_{0}^{r} n\left(r^{\prime}, t\right) d r^{\prime},
$$

where $f$ is the radar transmitter frequency and $r$ is the distance between the radar and fixed ground clutter targets. $r^{\prime}$ is the integrant. When $n$ is 1 , which is true when $P$ and $P_{\mathrm{w}}$ are zero or when the wave propagates in a vacuum,

$$
\varphi(r, t)=-\frac{2 r f}{c} 2 \pi=-\frac{2 r}{\lambda} 2 \pi
$$

where $\lambda \equiv c / f$ is the wavelength (here the minus sign is included based on convention). Because $2 r$ is the round-trip distance between the radar and ground target, $2 r / \lambda$ gives the round-trip distance in terms of the number of wavelength, and each wavelength corresponds to a change of phase of $2 \pi$. In the real atmosphere, the presence of atmosphere and water vapor modulates the value of $n$, hence affects the phase of EM waves returned to the radar. By examining phase difference between two ground clutters that serve as the backward scattering targets along the radar beams, mean refractivity index between those two ground targets can be estimated.

Equation (2.3) and the related discussion, however, show that the phase of the returned signal wraps around $2 \pi$ every half a wavelength (half due to the round trip), which is $5 \mathrm{~cm}$ for an S-band radar and 1.5 $\mathrm{cm}$ for X-band, making the use of phase problematic for the estimation of $n$. To solve this problem, Fabry et al. (1997) proposed using a reference map, which is made at the so-called reference time. Using this reference map, a reduction in phase wrapping is obtained by computing the phase change between the reference and observation times (Fabry et al., 1997). The reference time should be chosen when the temperature and humidity fields are nearly homogeneous over the region of refractivity observations so that the phase at the reference time can be determined with accuracy.

The phase change between measurement time $t_{\mathrm{m}}$ and reference time $t_{\text {ref }}$ for target at distance $r$ is given by

$$
\begin{aligned}
\delta \phi(r) & =\phi\left(r, t_{\mathrm{m}}\right)-\phi\left(r, t_{\mathrm{ref}}\right) \\
& =-\frac{4 \pi f}{c} \int_{0}^{r}\left[n\left(r^{\prime}, t_{\mathrm{m}}\right)-n\left(r^{\prime}, t_{\mathrm{ref}}\right)\right] d r^{\prime},
\end{aligned}
$$

where $\phi\left(r, t_{\mathrm{m}}\right)$ and $\phi\left(r, t_{\mathrm{ref}}\right)$ denote the currently measured and the reference phase values, respectively. An important point is that the phase change $\delta \phi$ wraps much less frequently than the phase measurement $\phi$ itself because it is based on the change in refractive index, which is much smaller than the actual refractive index. Of course, the drawback is that the method requires making reference phase measurements under sufficiently homogeneous conditions. Nevertheless, the phase-wrapping problem can be mitigated or reduced this way.

Again, we should emphasize that the refractive index change in Eq. (2.4) is integrated from the radar site to the range of ground target. By taking the phasechange difference (PCD) at two range values $R_{1}$ and $R_{2}$ along the same radial where $R_{2}>R_{1}$, we have

$$
\begin{aligned}
\Delta \phi\left(R_{1}, R_{2}\right) & =\delta \phi\left(R_{2}\right)-\delta \phi\left(R_{1}\right) \\
& =-\frac{4 \pi f}{c} \int_{R_{1}}^{R_{2}}\left[n\left(r^{\prime}, t_{\mathrm{m}}\right)-n\left(r^{\prime}, t_{\mathrm{ref}}\right)\right] d r^{\prime} \\
& =-\frac{4 \pi f}{c} \int_{R_{1}}^{R_{2}} \delta n\left(r^{\prime}\right) d r^{\prime},
\end{aligned}
$$

where $\delta n\left(r^{\prime}\right) \equiv n\left(r^{\prime}, t_{\mathrm{m}}\right)-n\left(r^{\prime}, t_{\mathrm{ref}}\right)$, i.e., the $\delta$ refers to the change between current and reference times, as in $\delta \phi$. From Eq. (2.5) we can obtain the mean refractivity index change over the range between $R_{1}$ and $R_{2}$ :

$$
\overline{\delta n\left(R_{1}, R_{2}\right)}=-\frac{c}{4 \pi f} \frac{\delta \phi\left(R_{2}\right)-\delta \phi\left(R_{1}\right)}{R_{2}-R_{1}} .
$$

To obtain the refractive index at the measurement time, the refractive index at the reference time must be known; it can be obtained through surface station measurements. In that case, the reference time should be chosen to be a time when the moisture distribution is relatively homogeneous so that the surface station network can properly characterize the field. Then, the refractive index at the measurement time is the sum of the refractive index at the reference time and the refractive index change. Using Eq. (2.1), the mean refractivity $N$ between the ground targets can then be obtained from the corresponding refractive index.

Two major limitations exist with the refractivitybased moisture measurement using the Fabry technique described above:

(1) To mitigate the severe phase-wrapping effect, phase measurements must be made at both the reference and measurement times. In addition to logistical problems of obtaining a reference map, the homogeneity assumption over the observed field is not justified under most conditions and certainly is difficult to validate.

(2) Because the actual locations of clutter targets are uncertain within the range volumes, it must be assumed that $R_{2}$ and $R_{1}$ are centered in their respective resolution volumes. This introduces the possibility of significant error in the finite difference operation in the final step of the algorithm Eq. (2.6). Even with 
the use of a reference field, phase wrapping can still occur, when

$$
\Delta \phi\left(R_{1}, R_{2}\right)=\left|\delta \phi\left(R_{2}\right)-\delta \phi\left(R_{1}\right)\right| \geqslant 2 \pi .
$$

Phase wrapping is more likely to occur when the distance between two ground targets is large and/or when the change in $n$ from the reference time is large. Generally, the observed PCD is $\bmod \left[\Delta \phi\left(R_{1}, R_{2}\right), 2 \pi\right]$. An unwrapping procedure is needed before the phasechange data can be used, unless the data analysis method is capable of unwrapping. Some of the inherent uncertainties associated with the phase measurements should be accounted for in the data assimilation process. This study will also examine some of these uncertainties.

\subsection{Generation of simulated observations}

To investigate issues associated with analyzing near-surface moisture using radar-based refractivity and phase measurements, simulated data are created and analyzed using a 2DVAR scheme. The simulated refractivity and phase change data are created using a radar emulator based on the equations in the previous subsection. Numerical experiments using simulated data are often called observation system simulation experiments (OSSEs). In an OSSE, a truth simulation of the atmosphere is created first, and simulated observations are then sampled from such a truth simulation. The model used to produce the truth field in this study was the Advanced Regional Pre- diction System (ARPS; Xue et al., 2003), a generalpurpose non-hydrostatic atmospheric model. A forecast of Xue and Martin (2006b) for a dryline convective initiation case from the 2002 International $\mathrm{H}_{2} \mathrm{O}$ Project (IHOP_2002) field experiment (Weckwerth et al., 2004) was used as the truth. This case was chosen because the rapid moisture change across the dryline and in time was good for testing the robustness of this system. The event occurred on 24 May 2002 over the southern Great Plains of the United States. For that forecast, the ARPS model was initialized using analysis data from the ARPS Data Analysis System (ADAS; Brewster, 1996) at 1800 UTC 24 May 2002, and the data were integrated for $6 \mathrm{~h}$. A $700-\mathrm{km} \times 400$ $\mathrm{km}$ model domain with a 1-km horizontal grid spacing was used (Fig. 1). For our simulation experiments, we assumed that the surface fields from their simulation were located at a constant height, i.e., over completely flat ground. These surface model fields were used for the simulation of $\Delta \phi\left(R_{1}, R_{2}\right)$, which were considered the raw measurement data.

An S-band radar $(\lambda=\mathrm{c} / \mathrm{f} \sim 0.1 \mathrm{~m})$ was assumed and placed at $x=230 \mathrm{~km}$ and $y=350 \mathrm{~km}$ of the domain (Fig. 1). We also assumed that the effective range of the $N$ measurements was within a $50-\mathrm{km}$ radius of the radar (i.e., the practical limit of such measurements due to the effect of the Earth's curvature). Two types of ground-target distributions were considered in this study: (1) Uniform (UFM) ground targets were distributed along the radar radials up to a $50-\mathrm{km}$
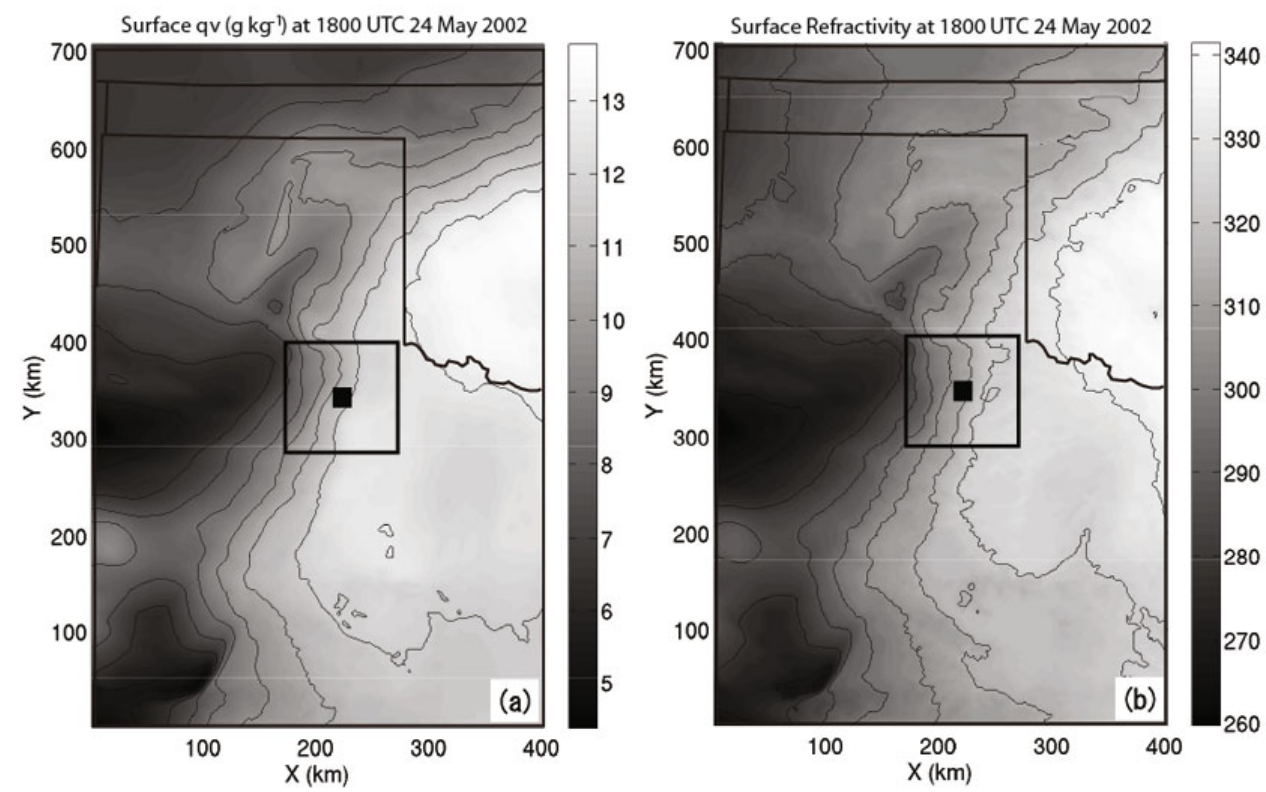

Fig. 1. (a) Surface water vapor and (b) simulated refractivity fields at 1800 UTC 24 May 2002, the reference time. The black square mark denotes the radar location and the open box denotes the domain shown in Fig. 2. 

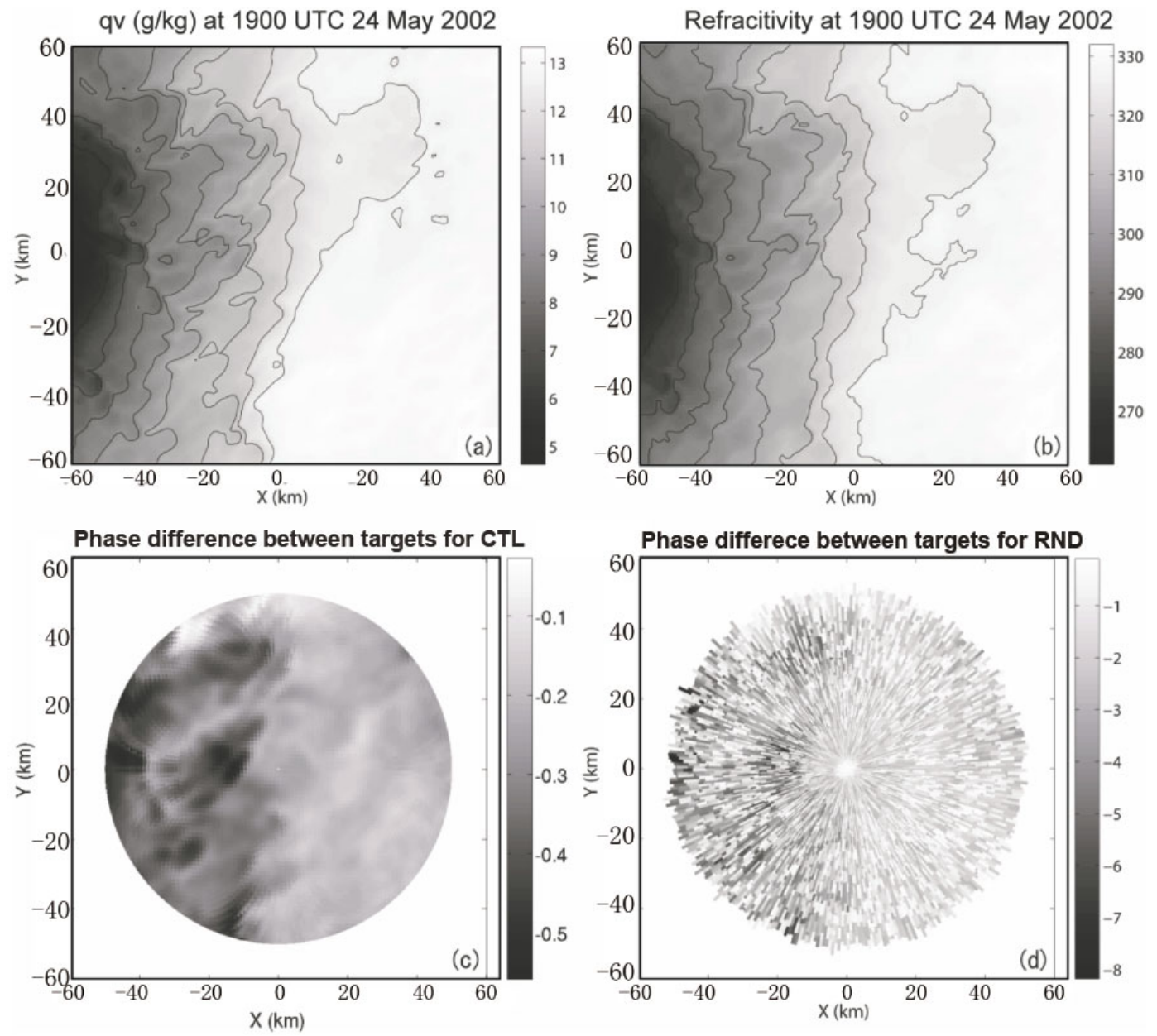

Fig. 2. Simulated observations at the surface at 1900 UTC 24 May 2002, $1 \mathrm{hr}$ after the reference time: (a) water vapor field, (b) the $N$ field, (c) the simulated phase difference between two targets for the case of uniform ground target case (hereafter UFM), and (d) the phase difference for the case of random target distribution, RND (see Table 1).

range with uniform spacing of $0.25 \mathrm{~km}$, equaling the gate spacing. (2) Random (RND) ground targets were distributed along the radials from the radar to $50 \mathrm{~km}$ with random spacing between $0.25 \mathrm{~km}$ and $4 \mathrm{~km}$.

For this study, the reference time was set at 1800 UTC 24 May 2002. The surface water vapor field for this time is shown in Fig. 1a. A distinct north-south dry line is evident in the surface field. The simulated refractivity field, $N$, at the same time, is given in Fig. 1b. The $N$ field has a quite similar pattern as the specific humidity field, $q_{v}$, given their strong interdependence.

Figure 2 shows the $q_{v}$, simulated $N$, and PCD fields at 1900 UTC (1 h after the reference time) derived from the truth simulation output. For the case of UFM with constant target spacing (Fig. 2c), the distribution of the simulated phase differences was similar to the simulated $N$ values, and the PCDs were not wrapped anywhere in the domain $(-0.6<\Delta \phi<0)$. For the RND case (Fig. 2d), the PCDs were proportional to the distance between targets, so the PCD field had an irregular pattern. In this case, phase wrapping often occurred where the distance between two targets was close to $4 \mathrm{~km}(-8<\Delta \phi<0)$. Initially, attempts were made to correct the phase wrapping in the variational analysis when additional mesonet-type moisture measurements were available. However, the phase measurements could not be unwrapped using this technique. Separate phase unwrapping had to be applied to the data before the variational analyses. In the experiments, either perfect phase-wrapping was assumed for the simulated data (first 8 experiments in Table 1 ), or the simulated data were subject to a practical phase-unwrapping procedure that had been developed and tested with real phase data (experiments UNW in Table 1), or the simulated data contained phasewrapping that had not been corrected (RND_WRP in Table 1). These experiments are discussed in section 
Table 1. List of experiments, their moisture analysis errors, and the observation error standard deviations. In the experiment names, UFM refers to experiments with uniform target spaces of $250 \mathrm{~m}$ and RND for randomly distributed targets with spacing between $250 \mathrm{~m}$ and $4 \mathrm{~km}$. UNW indicates the application of phase-unwrapping procedure to the phased-wrapped data. PE, OE and POE indicate added position errors, random observational errors, and both, respectively. WRP indicates the use of uncorrected phased wrapped data. RMSE is the root-mean square analysis error and ME the maximum absolute analysis error. 21 and 24 refer to the 2100 and 2400 UTC 24 May 2002 analysis times. In the last column are the error standard deviations (SDs) of the observations actually used, calculated against observations simulated the same way without adding error. Such SDs are used to specify observation error variance in $\boldsymbol{R}$ in the 2DVAR. In UFM, the observations have no error, and the $0.05^{\circ}$ is a small value used to specify $\boldsymbol{R}$ in the $2 \mathrm{DVAR}$.

\begin{tabular}{lcccccc}
\hline Experiments & $\begin{array}{c}\text { Target } \\
\text { distribution }\end{array}$ & $\begin{array}{c}\text { Position Errors } \\
( \pm 125 \mathrm{~m})\end{array}$ & $\begin{array}{c}\text { Obs. Errors } \\
\left(5^{\circ}\right)\end{array}$ & $\begin{array}{c}\text { RMSE } \\
\left(\mathrm{g} \mathrm{kg}^{-1}\right)\end{array}$ & $\begin{array}{c}\text { ME } \\
\left(\mathrm{g} \mathrm{kg}^{-1}\right)\end{array}$ & $\begin{array}{c}\text { Obs. Error } \\
\text { SDs }\left(^{\circ}\right)\end{array}$ \\
\hline UFM & Uniform & No & No & 0.031 & 0.201 & 0.05 \\
UFM_PE & Uniform & Yes & No & 0.039 & 0.432 & 6.348 \\
UFM_OE & Uniform & No & Yes & 0.078 & 0.487 & 5.017 \\
UFM_POE & Uniform & Yes & Yes & 0.083 & 0.523 & 8.071 \\
RND & Random & No & No & 0.036 & 0.217 & 0.129 \\
RND_PE & Random & Yes & No & 0.057 & 0.304 & 6.294 \\
RND_OE & Random & No & Yes & 0.049 & 0.225 & 4.994 \\
RND_POE & Random & Yes & Yes & 0.071 & 0.327 & 8.003 \\
RND_WRP & Random & No & No & 0.224 & 2.215 & 31.70 \\
UNW & Unwrapped & No & No & 0.137 & 0.957 & 20.92 \\
UNW_PE & Unwrapped & Yes & No & 0.140 & 0.991 & 21.01 \\
UNW_OE & Unwrapped & No & Yes & 0.154 & 1.112 & 22.48 \\
UNW_POE & Unwrapped & Yes & Yes & 0.147 & 1.033 & 22.83 \\
UNW_POE_21 & Unwrapped & Yes & Yes & 0.360 & 1.685 & 81.48 \\
UNW_POE_24 & Unwrapped & Yes & Yes & 0.245 & 2.065 & 42.25 \\
\hline
\end{tabular}

4 .

An important advantage with the variational analysis technique is the ability to properly account for errors in the analysis background and in the observations. Realistic observations should be simulated with expected error. The simulated data shown in Fig. 2 do not include added error. In this study, we simulated random observation errors that contained instrument (radar) measurement error, data representativeness error, and errors due to target position uncertainty. The random phase errors were assumed to have a Gaussian distribution with zero mean and a standard deviation of $5^{\circ}$, while the target position error was introduced by assuming the targets were always located at the center of the range gates (while the actual targets were not). These errors were simulated and added to the sampled phase, and the actual error standard deviations were calculated against error-free observations and were used to specify the observation error variance in the variational analysis. The observation errors were assumed to be uncorrelated.

\section{Two-dimensional variational analysis method}

\subsection{Methodology}

In this study, a two-dimensional variational (2DVAR) system was developed based on a univari- ate 3DVAR framework that was created for analyzing GPS slant-path water vapor data (Liu and Xue, 2006; Liu et al., 2007). For this study, the surface water vapor field was analyzed by assimilating the phase differences between two targets, i.e., $\Delta \phi\left(R_{1}, R_{2}\right)$.

The cost function of our 2DVAR system is defined as

$$
J\left(\boldsymbol{q}_{v}\right)=J_{\mathrm{b}}\left(\boldsymbol{q}_{v}\right)+J_{\phi}\left(\boldsymbol{q}_{v}\right),
$$

where

$$
\begin{gathered}
J_{\mathrm{b}}\left(\boldsymbol{q}_{v}\right)=\frac{1}{2}\left(\boldsymbol{q}_{v}-\boldsymbol{q}_{v, \mathrm{~b}}\right)^{\mathrm{T}} \boldsymbol{B}^{-1}\left(\boldsymbol{q}_{v}-\boldsymbol{q}_{v, \mathrm{~b}}\right), \\
J_{\boldsymbol{\phi}}\left(\boldsymbol{q}_{v}\right)=\frac{1}{2}\left[H\left(\boldsymbol{q}_{v}\right)-\Delta \boldsymbol{\phi}\right]^{\mathrm{T}} \boldsymbol{R}^{-1}\left[H\left(\boldsymbol{q}_{v}\right)-\Delta \boldsymbol{\phi}\right] .
\end{gathered}
$$

In Eq. (3.1), the cost function $J$ is composed of a background constraint term, $J_{\mathrm{b}}$, and phase difference observation term, $J_{\phi} . \quad J$ is minimized by using an optimization algorithm iteratively and the final analysis of the specific humidity $\boldsymbol{q}_{v}$ at the surface, $\boldsymbol{q}_{v, \text { a }}$, is the $\boldsymbol{q}_{v}$ that minimizes $J$. The corresponding background state vector is $\boldsymbol{q}_{v, \mathrm{~b}}$. The background term, $J_{\mathrm{b}}$, measures the departure of the control variable $\left(\boldsymbol{q}_{v}\right.$ in this case) from the background. Here $\boldsymbol{B}$ is the background error covariance matrix, which determines how the observational information is spread in space, and how the 
background is weighted in the analysis, relative to the observations. The above equations use the standard notation of data assimilation literature, as described in Ide et al. (1997). Additional information on variational data assimilation methodology, including the formation of cost function, can be found in Kalnay (2002).

The phase-difference observation term, $J_{\phi}$, represents the departure of the state $\boldsymbol{q}_{v}$ converted to the observed quantity through observational operator $H$, from the observations of the phase differences. In our case, the observation operator $H$ was based on Eq. (2.4), where $n$ was first calculated at the grid points. Line integrations were performed between ground targets, and $n$ was interpolated to the line segments to facilitate numerical integrations. This procedure is similar to that used by Liu and Xue (2006), where GPS slant-path water vapor was obtained by integrating along slant paths in a three-dimensional space.

The matrix $\boldsymbol{R}$ is the observation error covariance matrix for the phase difference between two targets, which is assumed to be diagonal in essentially all operational data assimilation systems under the assumption that observation errors are not correlated. This is due to difficulties in estimating observation error correlation and in inverting nondiagonal $\boldsymbol{R}$ matrix. In practice, most of the correlated errors (such as those related to radar beam elevation uncertainty, Bodine et al., 2011) can be decreased or removed through data thinning and/or bias correction (e.g., Harris and Kelly, 2001). For this study, the error variances, or the diagonal elements of matrix $\boldsymbol{R}$, whose determination was described in the last paragraph of section 2 , were small relative to the background error, the final analysis was therefore closer to the observations than to the background. As the first step to test a stand-alone variational analysis procedure to analyze the moisture field from refractivity phase-change observations, in the simulated data experiments we assumed that pressure and temperature were known, so that only the moisture field needed to be determined.

As in the work of Liu and Xue (2006) and Liu et al. (2007), the background error covariance matrix $\boldsymbol{B}$ was based on a spatial Gaussian correlation model that was isotropic or anisotropic and flow/field dependent. In this study, because the observation density was close to the grid resolution, the anisotropic, flowdependent, covariance model produced similar results to the isotropic covariance model. Therefore, only results from the isotropic covariance model are presented. Further, the effects of the spatial covariance were realized through spatial recursive filters, as proposed by Purser (1987) and Lorenc (1992), and the implementation followed Liu et al. (2007).

The following Gaussian function was used to model $\boldsymbol{B}$ (Huang, 2000):

$$
b_{i j}=\sigma_{\mathrm{b}}^{2} \exp \left[-\frac{1}{2}\left(\frac{r_{i j}}{L_{r}}\right)^{2}\right],
$$

where $\sigma_{\mathrm{b}}^{2}$ is the background error variance, which in our case was estimated based on the actual error of the background, given the truth field. $b_{i j}$ are the elements of $\boldsymbol{B}, r_{i j}$ is the distance between two grid points, $i$ and $j$, the grid indices. $L_{r}$ is the spatial decorrelation length scale. As pointed out by Kalnay (2002), for dense observations, $\boldsymbol{B}$ plays the role of observation information smoothing, and for sparse observations, $\boldsymbol{B}$ plays the role of observation information spreading. A larger $L_{r}$ in the above model tended to produce more smooth analyses, and a smaller $L_{r}$ tended to produce analyses that fit individual observations more tightly thereby retaining more small-scale structures present in the observations. In practice, the optimal decorrelation length scale depends on the observation density and the observation error level. It should also reflect the background error correlation. The optimal estimation and modeling of $\boldsymbol{B}$ represent a major research problem that continues to require active research (Kalnay, 2002).

\subsection{Experimental design}

In our experiments, 1800 UTC 24 May 2002 was chosen as the reference time for phase observations, and the moisture, temperature, and pressure fields were assumed known at this time. The analysis times 1900, 2100, and 2400 UTC were chosen; they allowed for the investigation of the sensitivity of analysis to time difference from the reference time. A larger increment increases the chance of phase wrapping.

The direct moisture output of the ARPS model is water vapor mixing ratio. The partial pressure of water vapor needed for the refractivity calculation was derived using the gas law $P_{\mathrm{w}}=q_{v} \rho_{\mathrm{d}} R_{\mathrm{v}} T$ where $\rho_{\mathrm{d}}$ is the dry air density and $R_{v}$ is the vapor gas constant. In our analysis, the reference refractivity, $N_{\text {ref }}$, was calculated from the $T, P$, and $q_{v}$ at 1800 UTC 24 May 2002. The background refractivity at each of the analysis times was calculated from $q_{v}$ at 1800 UTC and the $T$ and $P$ from the analysis time. Because of the time offset, the initial $q_{v}$ contains error and the error standard deviation (SD) can be calculated.

\section{Results of the single and perfect observa- tion experiments}

To variationally analyze the phase-change data, the observational operator $H$ was implemented into an ex- 
isting 3DVAR code, and the tangent linear and adjoint codes of the operator were developed. To verify the correctness of the minimization procedure of our $2 \mathrm{D}$ variational system and to help understand the behavior of the analysis system, an idealized singleobservation test was performed first. Further experiments using simulated phase-change data were performed, assuming no observational error or phase wrapping, or various forms of observation errors.

\subsection{Single observation test}

To perform a single-observation experiment, a single observation was created first. This single observation was a phase difference between two targets of 20 $\mathrm{km}$ apart, and it was simulated from the truth field between the targets. The decorrelation scale $L_{r}$ was specified as $3 \mathrm{~km}$. The observations were assumed to be perfect, which was equivalent to assuming that the error level of the background was extremely high. Therefore, the final analysis was expected to fit the observation closely.

The analysis increment from the single observation experiment is shown in Fig. 3. Because the observation was not a point measurement in this case but an integrated quantity along a path, the analysis increment exhibited a shape stretched in the direction of the path and spreading around the path, with the maximum influence radius being $<10 \mathrm{~km}$ from the path. The background value was -15.31 . The observation error SD was specified to be a very small $0.05^{\circ}$ in the 2DVAR. The analyzed phase difference was -18.928 , very close to the observed value of -18.93 as expected.

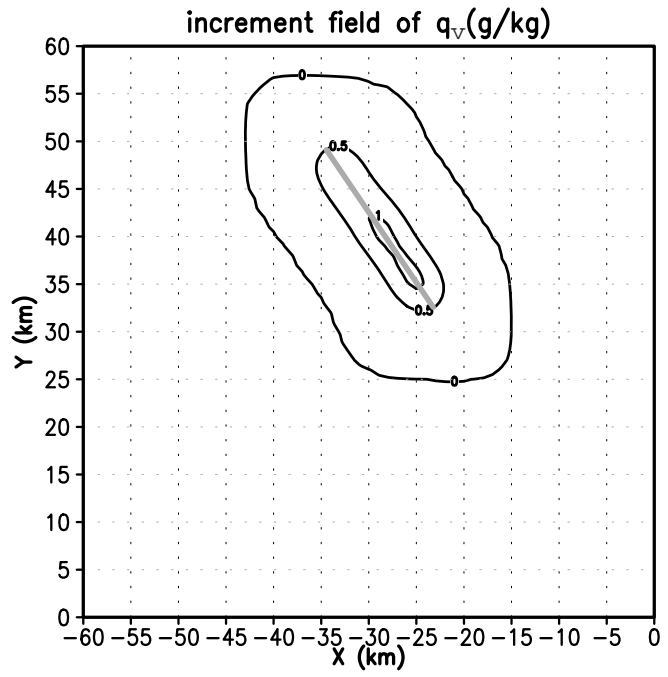

Fig. 3. Analysis increment of moisture from the single observation experiment. The contour intervals are $0.5 \mathrm{~g} \mathrm{~kg}^{-1}$ and outermost contour has a value of zero. Gray straight line denotes the path between two targets $20 \mathrm{~km}$ apart.

\subsection{Perfect observation experiments}

To analyze the full $2 \mathrm{D}$ water vapor field from simulated phase difference observations, two perfect observation experiments (UFM and RND in Table 1) were performed. These experiments allowed us to examine how well the analyzed moisture field was able to fit the truth, in the case of very accurate observations. Because no observation error was associated with the data, we specified a very low $0.05^{\circ}$ standard deviation for $\boldsymbol{R}$. The de-correlation scale $L_{r}$ of the error covariance model was $3 \mathrm{~km}$. The sensitivity of the analysis to the value of the de-correlation scale was examined.

The first experiment was UFM (Table 1), where the distribution of the ground targets was uniform in the radial direction (see section 2 ). The second experiment, RND, was the same as UFM, except that the ground targets were randomly spaced between $250 \mathrm{~m}$ and $4 \mathrm{~km}$ (Table 1 ), which were more realistic.

The 2DVAR cost function and the norm of the cost-function gradient as function of the number of iterations during the minimization procedure for experiment UFM were plotted (Fig. 4). Significant reductions occur in both quantities occurred during the first 20 iterations. Afterward, the cost function remained relatively flat while the gradient norm continued to decrease over the next 50 iterations or so. In all cases, the minimization algorithm was run for at least 100 iterations, which appeared to have been sufficient for the desired accuracy. The cost function and the gradient no longer decrease after 95 iterations (Fig. 4).

The analyzed water vapor mixing ratio fields for UFM and RND at 1900 UTC 24 May 2004, $1 \mathrm{hr}$ after the reference time, are shown in Figs. 5a and b, respectively. Both analyses were very close to the truth field where phase-change observations were available. The observations were confined within the $50-\mathrm{km}$ range of the radar, located at the center of plotting domain. The RMSE and the maximum error (ME) between the analysis and truth fields are presented in Table 1. The RMSE and ME were calculated only within the $50-\mathrm{km}$ radar range. The RMSEs for UFM and RND were 0.031 and $0.036 \mathrm{~g} \mathrm{~kg}^{-1}$, respectively, which indicate very good analyses in both cases. The ME for UFM and RND were 0.201 and $0.217 \mathrm{~g} \mathrm{~kg}^{-1}$, respectively. For a reference, the RMSE between the background and truth fields was $1.06 \mathrm{~g} \mathrm{~kg}^{-1}$; therefore, the error was reduced by more than one order of magnitude. These results show that the 2DVAR system works very well to analyze the moisture field from the phase-change data when the influence of phase wrapping and observational errors could be negligible. UFM had smaller errors than RND because of its higher, uniform observation density. 

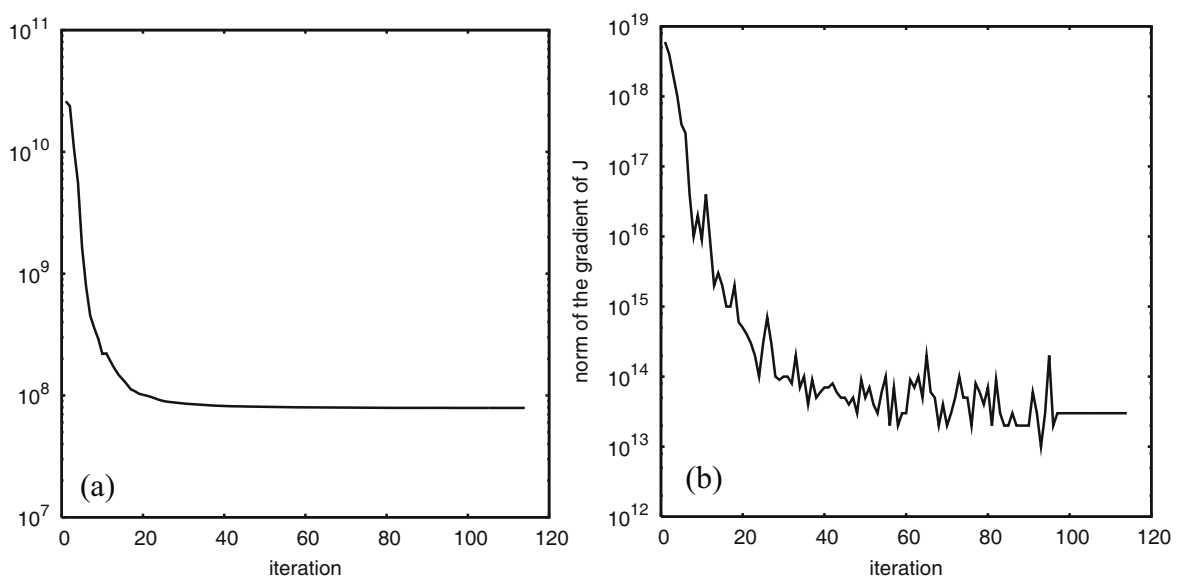

Fig. 4. (a) The variation of the cost function $J$ and (b) the norm of the gradient $\nabla J$, with the number of iterations during the minimization procedure for experiment UFM.
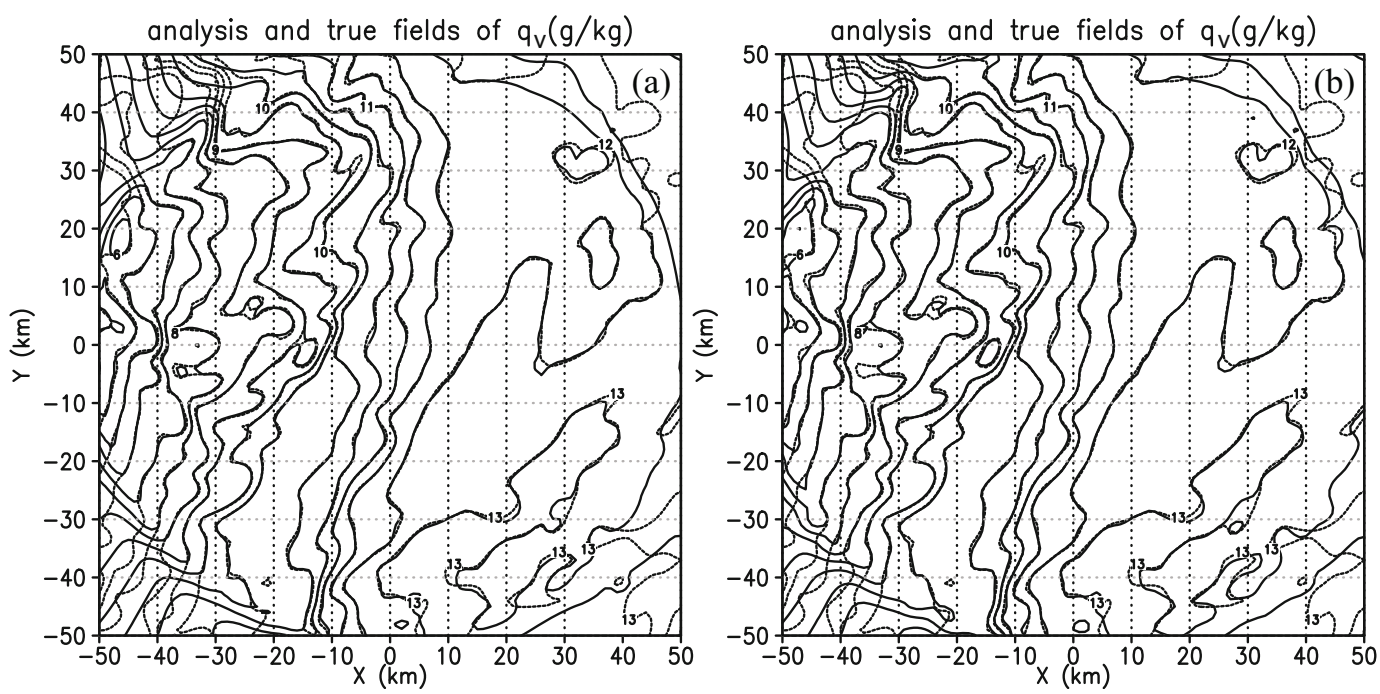

Fig. 5. 2D analysis of water vapor fields (sold lines) at 1900 UTC 24 May 2002 (a) from UFM and (b) from RND. Dashed lines are for the truth field. The contour interval is $0.5 \mathrm{~g} \mathrm{~kg}^{-1}$.

\subsection{Sensitivity to decorrelation scale}

In the absence of reliable error statistics, the decorrelation scales used in background error correlation models, as described by Eq. (3.3), were often empirically determined. Optimal decorrelation scales often exist for a particular observational network and a particular observation error level. As discussed earlier, these scales control the spatial extent over which observation increments are spread, the smoothness of the analysis, and how well the analysis fits the observations. A fixed value of $3 \mathrm{~km}$ was used for $L_{r}$ in experiments UFM and RND as reported earlier. The overall RMSE $\left(\mathrm{g} \mathrm{kg}^{-1}\right)$ of the analyses as a function of the decorrelation length $L_{r}$ when experiments UFM and RND as well as other experiments to be discussed later were rerun using $L_{r}$ values ranging from $1 \mathrm{~km}$ to 10 km (Fig. 6). For UFM and RND, the perfect observation cases, the optimal decorrelation length was $\sim 3$ $\mathrm{km}$.

\section{Results of theimperfect observation experi- ments}

\subsection{Impact of target position error}

For the S-band radar assumed in this study, the range resolution was $250 \mathrm{~m}$, which is the range resolution of the operational Weather Surveillance Radar 1988D (WSR-88D) radars. Because of the finite range resolution, it was impossible to know the exact positions of the ground targets within each range gate. The resulting ambiguity created target position errors because the actual phase measurement corresponded 


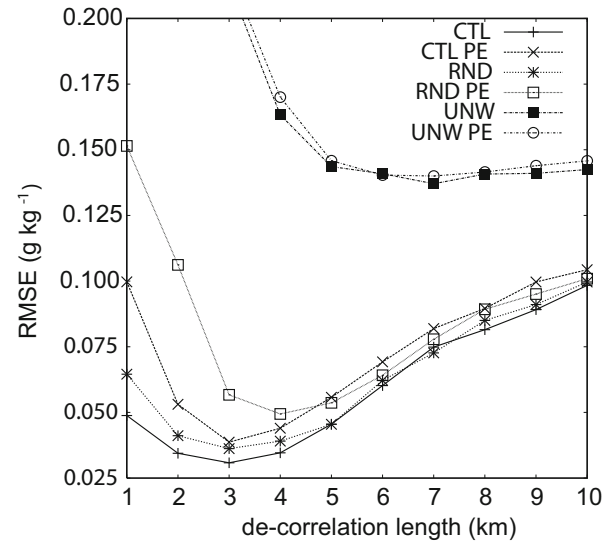

Fig. 6. The overall RMSE $\left(\mathrm{g} \mathrm{kg}^{-1}\right)$ between analysis and truth field as a function of the decorrelation length $L_{r}$.

to the mean target location (weighted by reflectivity) within the gate, not the physical center of the gate, which was assumed to be the target location in that gate. Hence, errors associated with target position uncertainty should be taken into account when estimating the phase measurement error, and the relative error was larger for smaller target distances.

To examine the impact of target position error, simulated error was added to the phase measurements in the next set of experiments with uniform and random spacing. The added target position error was assumed to have the form $r_{\text {target }}=r_{\text {gate }}+\alpha 250 / 2$, where $\alpha$ was a random number between -1 and 1 with a uniform distribution. Here $r_{\text {target }}$ is the range of actual ground target, but the phase-difference observations were assumed to correspond to $r_{\text {gate }}$. The two experiments performed were called UFM_PE and RND_PE, where PE indicates position error. These experiments were the same as experiments UFM and RND, except that range errors were included when simulating the phase-difference observations.

As shown in Table 1, the RMSEs for UFM_PE and RND_PE were 0.039 and $0.057 \mathrm{~g} \mathrm{~kg}^{-1}$, respectively, and the MEs were 0.432 and $0.304 \mathrm{~g} \mathrm{~kg}^{-1}$, respectively. As expected, the MEs for both cases were increased due to the added target position error but the increases were not large in absolute value. This is not surprising, because the range spacing of $250 \mathrm{~m}$ was much smaller than the $1-\mathrm{km}$ spacing of the analysis grid. The sensitivity of UFM_PE and RND_PE to the decorrelation scale is shown in Fig. 6. The optimal de-correlation length remained $3 \mathrm{~km}$ for UFM_PE and increased to $4 \mathrm{~km}$ for RND_PE. Larger RMSE differences between the error and no-error cases were found when a small decorrelation scale was used. For example, the RMSE was $\sim 0.15 \mathrm{~g} \mathrm{~kg}^{-1}$ for RND_PE versus $\sim 0.1 \mathrm{~g} \mathrm{~kg}^{-1}$ for RND when $L_{r}$ was $1 \mathrm{~km}$. In the presence of observation error, the spatial covariance acted to smooth out nonsystematic observation errors when more observations were involved in the analysis of grid-point values. Without this smoothing effect (i.e., $L_{r}$ near the grid interval), the analysis tended to be noisy, contaminated by the errors from individual observations. When an optimal decorrelation scale was used, and/or when the average target spacing was significantly larger than the range resolution, the impact of the target position uncertainty and the associated phase-measurement error on the water vapor analysis was minimal.

\subsection{Impact of random observational error}

The experiments presented thus far did not considered typical random observational error, which was caused by radar phase-measurement error, ground target motion, radar beam height uncertainty, and changes in the vertical gradient of refractivity (Bodine et al., 2011). Such errors were properly simulated by adding sampled errors to the raw phase measurements, from which the PCD data were derived with or without phase wrapping. The added phase errors had $1^{\circ}, 2^{\circ}, 5^{\circ}$ or $10^{\circ}$ standard deviations, with a Gaussian distribution in four sets of experiment, but we focused on the results of $5^{\circ}$ error. These experiments were named UFM_OE and RND_OE (Table 1). Additionally, UFM_POE and RND_POE further included position-related error, as simulated earlier.

The analysis error statistics for UFM_OE, RND_OE, UFM_POE and RND_POE are shown in Table 1 . The RMSEs for them were $0.092,0.049,0.083$, and $0.071 \mathrm{~g} \mathrm{~kg}^{-1}$, respectively. These RMSEs increased compared to the corresponding no-error cases, but the errors in absolute terms remained small. The analysis fields for UFM_POE and RND_POE at 1900 UTC 24 May 2004 are provided in Fig. 7. Both analysis fields remained rather close to the truth field in general. The analysis of RND_POE showed noticeable differences in small-scale structures from the truth. This is the case because the phase-difference measurements in RND_POE spanned target spacing ranging from $250 \mathrm{~m}$ to $4 \mathrm{~km}$. Errors along the path between two adjacent ground targets effectively had the same sign; they did not cancel each other as is possible in the UFM_POE case. For this type of error, more ground targets reduced errors, contrary to the position error type.

The RMSEs of the analysis fields as a function of the absolute phase errors for experiments UFM_OE, RND_OE, UFM_POE and RND_POE are shown in Fig. 8. For the uniform target cases (UFM_OE and UFM_POE), the RMSE increased with the observation error more rapidly than with the nonuniform tar- 

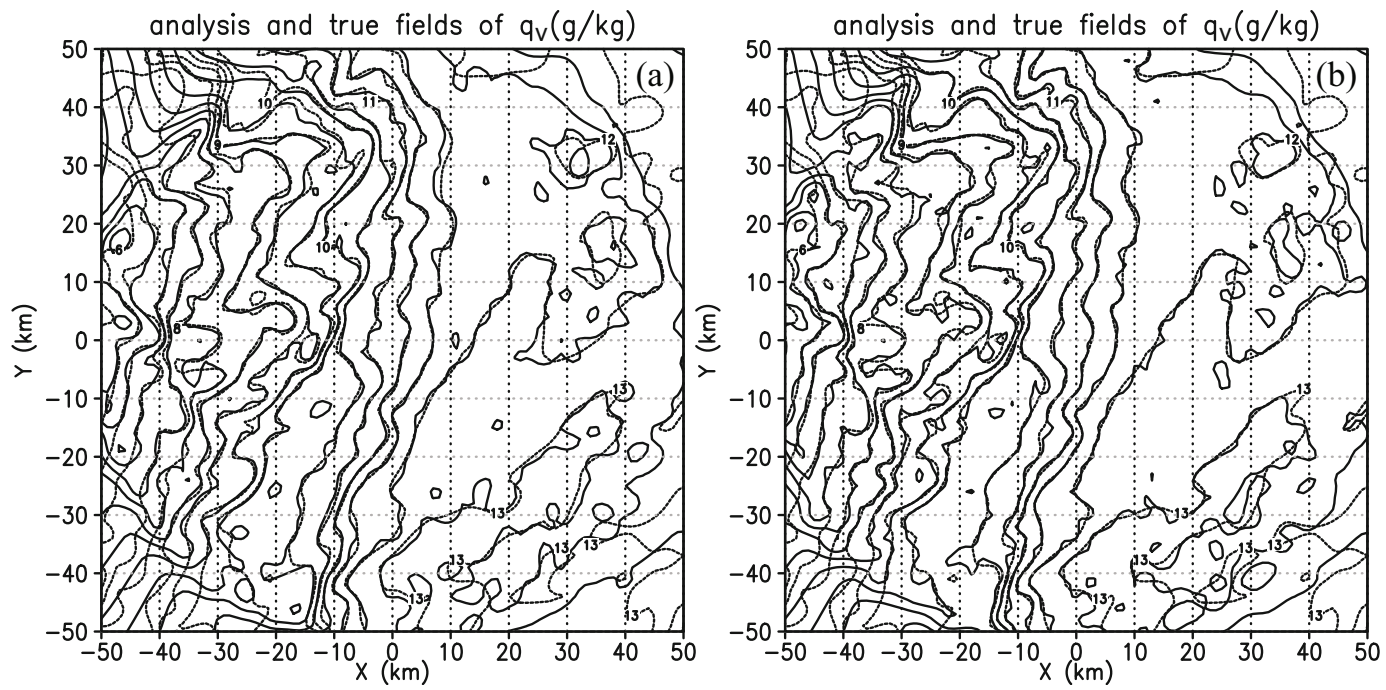

Fig. 7. Analyses of surface water vapor fields (sold lines) at 1900 UTC 24 May 2002 (a) from UFM_POE and (b) from RND_POE. Both include observational error with $5^{\circ}$ standard deviation and target position error. Dashed lines denote the truth field. The contour interval is $0.5 \mathrm{~g} \mathrm{~kg}^{-1}$.

get cases; in the latter case, the errors were spread over longer paths so that the total error accumulation was less. The impact of additional position error was generally small because the RMSEs for the OE and POE cases were very close. The RMSEs for the POE cases were not always larger than the corresponding OE cases, and any differences among them were probably insignificant.

\subsection{Impact of phase wrapping error}

The experiments presented thus far assumed that the data were not subject to phase wrapping. In the actual observations, however, phase wrapping did oc-

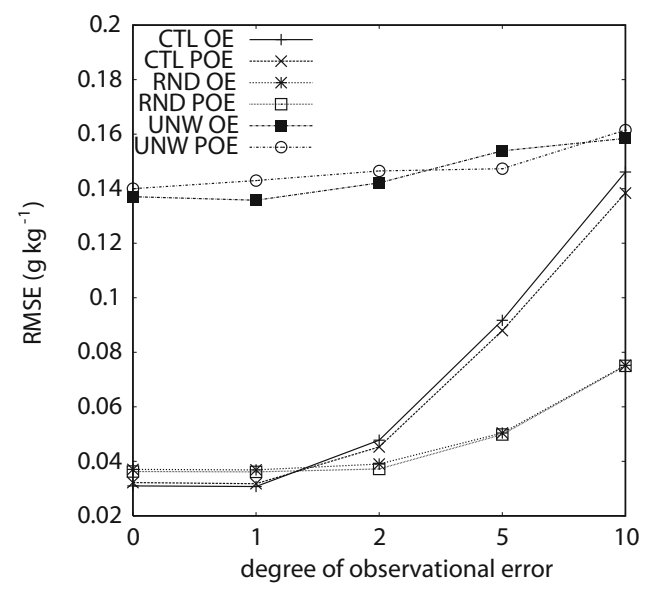

Fig. 8. The overall RMSEs $\left(\mathrm{g} \mathrm{kg}^{-1}\right)$ of analysis as a function of absolute phase observational error standard deviation with a Gaussian distribution, for the six experiments. cur (Fig. 2d). As discussed earlier, phase wrapping is more likely to occur when the distance between two targets is large and/or when the refractivity change from the reference time is large. In our experiments, only the RND case had a phase-wrapping issue. To include this effect, experiment RND_WRP was performed, which was the same as RND except that the original simulated phase-difference observations were phased wrapped when they exceeded $2 \pi$ (i.e., the observations analyzed are $\bmod \left[\Delta \phi\left(R_{1}, R_{2}\right), 2 \pi\right]$, the remainder of $\left.\Delta \phi\left(R_{1}, R_{2}\right) /(2 \pi)\right)$. No effort was made to unwrap such observations, thus some of the observations used may have contained very large errors.

The moisture analysis for RND_WRP at 1900 UTC 24 May 2004 is shown in Fig. 9a. The analysis field exhibited erroneous values where phase wrapping occurred (c.f., Fig. 2d). The RMSE and ME for RND_WRP were $0.224 \mathrm{~g} \mathrm{~kg}^{-1}$ and $2.215 \mathrm{~g} \mathrm{~kg}^{-1}$, respectively. The ME was very large because the phasewrapped observations had very large errors. In practice, a quality control procedure might be useful in eliminating such erroneous data.

Because the analysis process was unable to solve the variational phase-wrapping problem, even when additional mesonet moisture observations were available (not shown), a phase-unwrapping procedure was applied to the raw data first. The procedure described in Cheong et al. (2008) for real refractivity data processing was used here. The procedure first calculated the mean of phase-wrapping rate for the entire map and subtracts a synthetic mean phasewrapping map from the entire map. Then, the proce- 

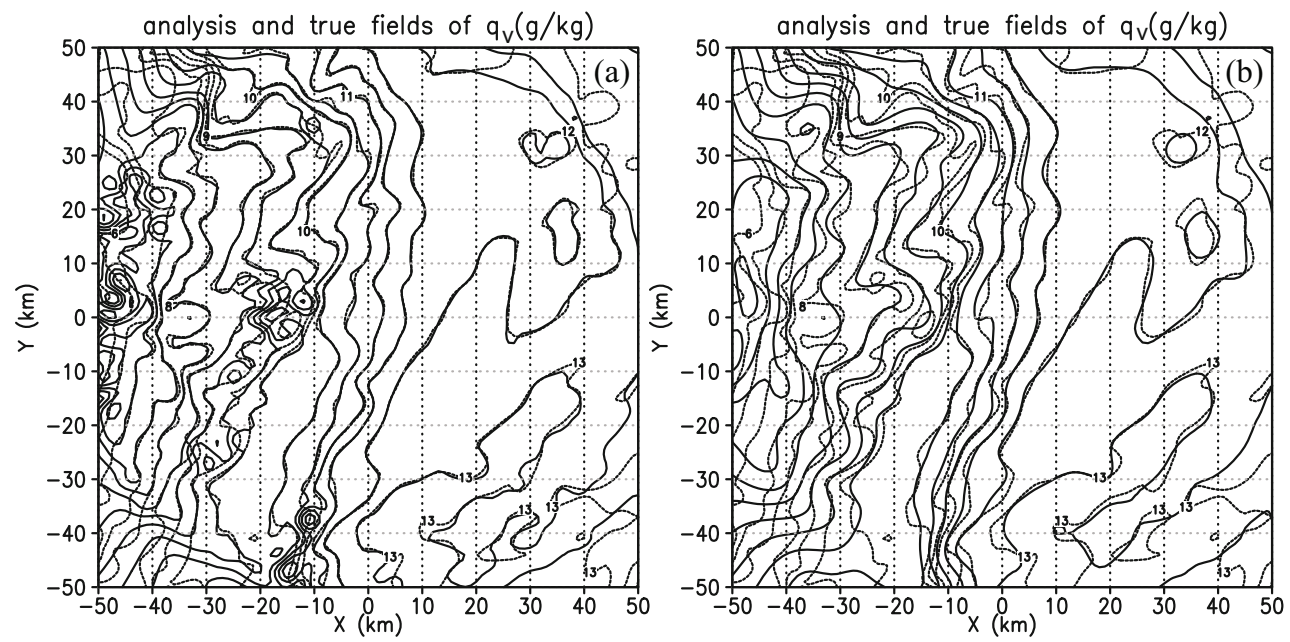

Fig. 9. 2DVAR analysis of surface water vapor mixing ratio (sold lines) at 1900 UTC 24 May 2002 from (a) RND_WRP and (b) UNW. Dashed lines denote the truth field. The contour interval is 0.5 $\mathrm{g} \mathrm{kg}^{-1}$.

dure smoothes and interpolates the perturbation component using two convolutions (one across range and the other across azimuth) to achieve an equivalent 2D filtering effect. The weights are a function of range and azimuth to preserve a same spatial coverage of $\sim 4 \times 4$ $\mathrm{km}$. This perturbation phase map was unwrapped on a radial-by-radial basis by the abrupt phase change from $-\pi$ to $+\pi$. Finally, the mean phase-wrapping rate is accumulated and added to the perturbation phase component to derive the unwrapped phase for each range cell.

Experiment UNW was performed, which was the same as RND_WRP except that phase 'unwrapping' was applied to the raw observations first. Figure $9 \mathrm{~b}$ shows the analysis field for UNW at 1900 UTC 24 May 2002. The unrealistic values in Fig. 9a are largely gone. However, because the unwrapping process involves spatial averaging, the analysis field appeared smoother than the truth. The RMSE and ME of UNW were 0.137 and $0.957 \mathrm{~g} \mathrm{~kg}^{-1}$, respectively; even though they were larger than the RND case, the error was still considered low for low-level moisture.

The next three experiments, UNW_PE, UNW_OE, and UNW_POE (Table 1) were the same as UNW, except for the addition of position error, random error, and both, respectively. These error sources generally increased the RMSEs and MEs slightly, but the increases were smaller compared to the error increase due to phase wrapping and unwrapping (compare UNW and RND). The impact of phase wrapping and unwrapping was generally larger than either of those two error sources (Table 1).

With phase unwrapping, the optimal decorrelation scale of the 2DVAR analysis was increased to 7 $\mathrm{km}$ from the earlier $3 \mathrm{~km}$ (see Fig. 6 for UNW and
UNW_PE). The higher optimal decorrelation scale was because the original simulated observations were interpolated and smoothed in the unwrapping procedure, resulting in a lower effective resolution of the final observations. For all of the analyses presented in this paper, UNW experiments used the optimal 7$\mathrm{km}$ decorrelation scale. Figure 10a shows the analysis field for experiment UNW_POE, which is the same as UNW_PE except for the added random observational errors. The analyzed field again appeared smoother as in UNW (see Fig. 9b), with its RMSE and ME being 0.147 and $1.033 \mathrm{~g} \mathrm{~kg}^{-1}$, respectively, similar to those of UNW. In a sense, experiment UNW_POE included the main sources of expected error associated with phase-change measurements between ground targets. By applying a phase-unwrapping procedure that had been proven effective to real measurements, rather accurate surface moisture analysis was obtained using a variational procedure developed in this study. Apparently, the analysis was not very sensitive to the expected data error for the situations considered. In the next subsection, the impact of the time difference from the reference was examined.

\subsection{Sensitivity to the reference time differ- ence}

All of the experiments presented so far used simulated observations and the analyses were conducted using 1900 UTC 24 May 2002, 1 hr after the reference time. To investigate the sensitivity to longer referencetime differences (for which more phase wrapping might occur), we repeated experiment UNW_POE at 2100 UTC and 2400 UTC (experiments UNW_POE_21 and UNW_POE_24), three and six hours after the reference time, respectively. The analysis fields for these two ex- 

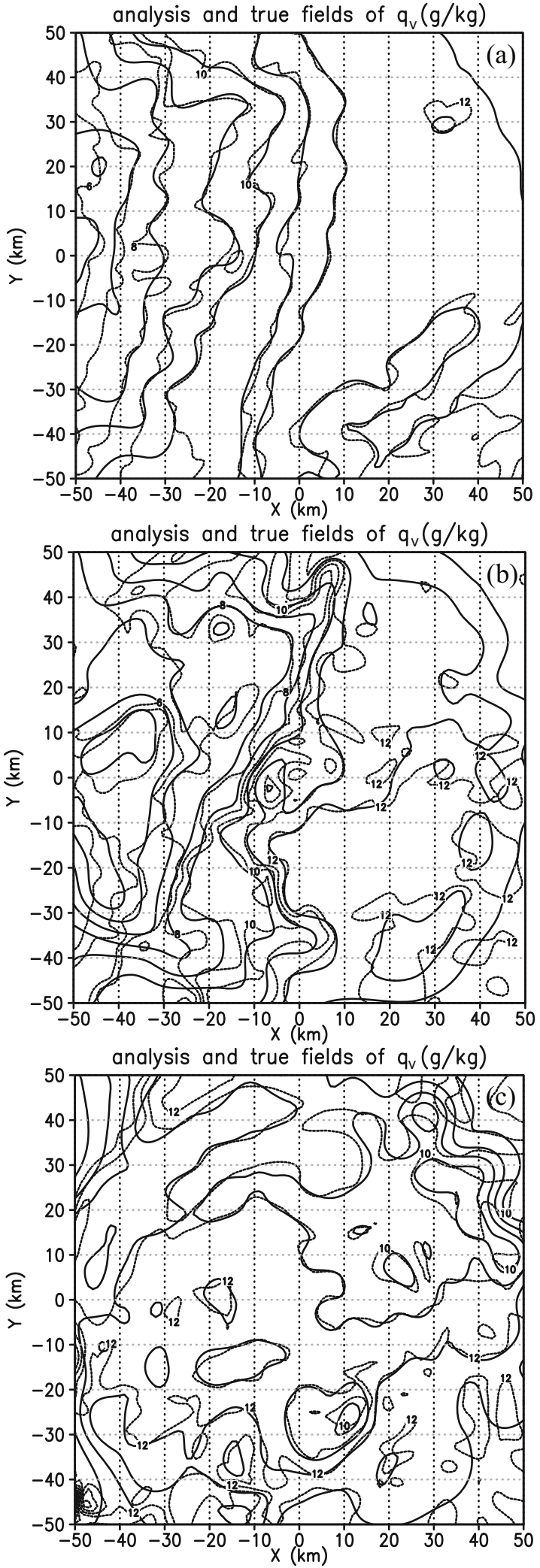

Fig. 10. 2DVAR analysis of surface water vapor fields (sold lines) from UNW_POE at (a) 1900 UTC, (b) 2100 UTC, and (c) 2400 UTC 24 May 2002. Dashed lines denote the truth field. The contour interval is $1.0 \mathrm{~g} \mathrm{~kg}^{-1}$. periments are shown in Figs. 10b and c. By these times, the model simulations developed fine-scale boundary layer convective eddies and rolls (Xue and Martin, 2006a), and convection was initiated along the dryline around 2100 UTC. By 2400 UTC, the main dry line retreated westward and was located mostly outside the plotted domain (Fig. 10c). The moisture fields exhibited more small-scale structures than at 1900 UTC. For this reason, the moisture analysis RMSEs for them were somewhat larger than those with the earlier time, at 0.360 and $0.245 \mathrm{~g} \mathrm{~kg}^{-1}$, respectively (Table 1). Figures $10 \mathrm{~b}$ and $\mathrm{c}$ show that the analyzed contours still matched the truth reasonably well. In these two cases, the background $q_{v}$ used in the cost function and for calculating the reference phase differences was more different from the truth at these times than at 1900 UTC, with the RMSE between the background and truth fields being $2.32 \mathrm{~g} \mathrm{~kg}^{-1}$ for 2100 UTC and $1.75 \mathrm{~g} \mathrm{~kg}^{-1}$ for 2400 UTC. Despite more occurrences of phase wrapping (not shown), the analysis quality remained reasonable, indicating the effectiveness of the phase-unwrapping procedure.

\section{Conclusions}

This study examined the ability to accurately analyze surface moisture fields at a high spatial resolution, from electromagnetic wave phase difference data that can be measured by ground-based weather radars between ground targets aligned along the radar beams. The measurement was based on the principle that the phase of the backscattered radar signal from ground targets was related to the path-integrated refractive index, which was strongly linked to water vapor (Fabry et al., 1997). To reduce the phase-wrapping problem, a known reference state was employed, as originally proposed by Fabry et al. (1997), so that only incremental phase differences between ground targets had to be calculated, which were less susceptible to phase wrapping. Two sets of experiment with simulated observations were performed, one assuming a uniform ground target spacing of $250 \mathrm{~m}$ along the radar radials, and one assuming randomly varying nonuniform spacing between $250 \mathrm{~m}$ and $4 \mathrm{~km}$. A 1-km resolution numerical simulation of a dryline case was used as the truth, which was sampled using a realistic radar simulator to produce refractivity-related phase change difference observations between ground targets. Several sources of observation error were considered and their impacts examined, including errors due to ground target position uncertainty, and typical random errors associated with radar phase measurements. Gross error due to phase wrapping as well as error associated with spatial averaging and smoothing in the phase unwrapping 
procedure was also considered.

A 2DVAR analysis system was developed for analyzing the surface water vapor from the phase-change observations. An observation operator and its adjoint were implemented within the 2DVAR system that involves numerical integration along the radar beams between targets. Analysis experiments were conducted with the simulated observations with different error characteristics, and analysis errors were calculated against the truth fields. The background and observation error variances were specified based on their actual error statistics calculated against the truth. The spatial covariance of the boundary error was modeled using a Gaussian function, and the optimal background error decorrelation scale was determined through numerical experimentations. The results are summarized as follows:

(1) The surface moisture field analyses produced by our 2DVAR system from the phase change data captures the detailed structure of the moisture field rather accurately, when no phase-wrapping problem exists in the data.

(2) Experiments with data suffering from ground target position ambiguity (uncertain within one range gate) show that such errors affect the analysis quality only slightly, and the effect is smaller when the target spacing is large relative to the gate spacing.

(3) Experiments with data containing random phase errors with standard deviations of up to $10^{\circ}$ (results with $5^{\circ}$ error were focused on) show that the impact of such errors are also relatively small. Even in the presence of both target position uncertainty and random measurement errors, the RMSEs of the analysis were generally $<0.1 \mathrm{~g} \mathrm{~kg}^{-1}$, while the maximum error was generally $<0.5 \mathrm{~g} \mathrm{~kg}^{-1}$.

(4) Our 2DVAR system failed to produce reasonable surface moisture analysis when the observations included phase wrapping. When the data were first unwrapped in a separate pre-processing step, quality analyses were produced. The unwrapping process involves spatial interpolation and smoothing of the original phase difference data, hence reducing the effective resolution of the observations and the resulting moisture field loses some of the fine scale details in the truth field, and the RMSEs are usually between 0.1 and $0.21 \mathrm{~g} \mathrm{~kg}^{-1}$, but the maximum error can exceed $1 \mathrm{~g} \mathrm{~kg}^{-1}$. The optimal analysis decorrelation scale is also larger.

(5) When the difference between the analysis and reference times is larger, more phase wrapping tends to occur. The phase-unwrapping procedure applied was still effective, and the resultant analyses were of slightly poorer quality, but the RMSEs remained below $0.5 \mathrm{~g} \mathrm{~kg}^{-1}$. This suggests that the technique us- ing a reference time and state in combination with a phase-unwrapping procedure can be successfully applied, as long as the state at the reference time can be estimated with sufficient accuracy.

Finally, we note that, as an initial effort to variationally analyze moisture field from radar phase change data, we assume that the temperature and pressure fields are known. In general, refractivity is most sensitive to moisture. But in real-world problems, temperature and pressure fields also need to be analyzed; this is the main limitation of this study. In a 2DVAR or 3DVAR system without reliable, flowdependent, cross-covariance of background error, the adjustment to temperature and pressure from the refractivity data mainly depend on the sensitivity of refractivity to these variables and their background values. In the future, we plan to use multivariate data assimilation methods, such as the ensemble Kalman filter (EnKF; Evensen, 2003, 2006), where flow-dependent background covariance derived from the ensemble is used to analyze all fields simultaneously. The 2D and $3 \mathrm{D}$ variational methods are useful for objective analyses of surface moisture fields for weather forecasting and diagnostic purposes and for initializing numerical weather prediction models when time constraints are severe. The flow-dependent error covariance from EnKF also helps to optimally determine the vertical spread of surface moisture observation information. We also realize that in the real world, the refractivity observation can be more complicated than considered in this study, and certain aspects of error, such as those related to representativeness, can be very difficult to quantify (Bodine et al., 2011). Further research is needed in these areas, as well as demonstration of the impact of real refractivity observations for real cases.

Acknowledgements. Drs. Michihiro TESHIBA, Ming HU and Yunheng WANG provided great support during this work. The authors also wish to thank Dr. Yoshi SASAKI for many helpful suggestions. K. SHIMOSE was supported by the Research Fellowship for Young Scientists by the Japan Society for the Promotion of Science. This work was primarily supported by the National Science Foundation (Grant No. AGS-0750790). M. XUE was also supported by the NSF grants (Grant Nos. AGS0802888, OCI-0905040, AGS-0941491, AGS-1046171, and AGS-1046081).

\section{REFERENCES}

Anthes, R. A., and Coauthors, 2008: The COSMIC/ FORMOSAT-3 Mission: Early results. Bull. Amer. Meteor. Soc., 89, 313-333.

Bean, B., R. and E. J. Dutton, 1968: Radio Meteorology. Dover Publications, 435pp. 
Bodine, D., and Coauthors, 2011: Understanding radar refractivity: Sources of uncertainty. J. Atmos. Oceanic Technol., 50, 2543-2560.

Brewster, K., 1996: Application of a Bratseth analysis scheme including Doppler radar data. Preprints, 15th Conf. Weather Analysis Forecasting, Norfolk, VA, Amer. Meteor. Soc., 92-95.

Brock, F. V., K. C. Crawford, R. L. Elliott, G. W. Cuperus, S. J. Stadler, H. L. Johnson, and M. D. Eilts, 1995: The Oklahoma Mesonet: A technical overview. J. Atmos. Oceanic Technol., 12, 5-19.

Cheong, B. L., R. D. Palmer, C. Curtis, T.-Y. Yu, D. S. Zrnic, and D. Forsyth, 2008: Refractivity retrieval using the phased array radar: First results and potential for multi-mission operation. IEEE Trans. Geosci. Remote Sens., 46, 2527-2537.

Droegemeier, K. K., and Coauthors, 2000: Hydrological aspects of weather prediction and flood warnings: Report of the ninth prospectus development team of the U.S. weather research program. Bull. Amer. Meteor. Soc., 81, 2665-2680.

Emanuel, K., and Coauthors, 1995: Report of the first prospectus development team of the U.S. weather research program to NOAA and the NSF. Bull. Amer. Meteor. Soc., 76, 1194-1208.

Evensen, G., 2003: The ensemble Kalman filter: Theoretical formulation and practical implementation. Ocean Dynamics, 53, 343-367.

Evensen, G., 2006: Data Assimilation: The Ensemble Kalman Filter. Springer, 280pp.

Fabry, F., C. Frush, I. Zawadzki, and A. Kilambi, 1997: On the extraction of near-surface index of refraction using radar phase measurements from ground targets. J. Atmos. Oceanic Technol., 14, 978-987.

Fritsch, J. M., and Coauthors, 1998: Quantitative precipitation forecasting: Report of the eighth prospectus development team, U.S. Weather Research Program. Bull. Amer. Meteor. Soc., 79, 285-299.

Harris, B. A., and G. Kelly, 2001: A satellite radiancebias correction scheme for data assimilation. Quart. J. Roy. Meteor. Soc., 127, 1453-1468.

Huang, X.-Y., 2000: Variational analysis using spatial filters. Mon. Wea. Rev., 128, 2588-2600.

Ide, K., P. Courtier, M. Ghil, and A. Lorenc, 1997: Unified notation for data assimilation: Operational, sequential and variational. J. Meteor. Soc. Japan, 75, 181-189.

Kalnay, E., 2002: Atmospheric Modeling, Data Assimilation, and Predictability. Cambridge University Press, $341 \mathrm{pp}$.

Liu, H., and M. Xue, 2006: Retrieval of moisture from slant-path water vapor observations of a hypothetical GPS network using a three-dimensional variational scheme with anisotropic background error.
Mon. Wea. Rev., 134, 933-949.

Liu, H., M. Xue, R. J. Purser, and D. F. Parrish, 2007: Retrieval of moisture from simulated GPS slantpath water vapor observations using 3DVAR with anisotropic recursive filters. Mon. Wea. Rev., 135, 1506-1521.

Lord, S. J., E. Kalnay, R. Daley, G. D. Emmitt, and R. Atlas, 1997: Using OSSEs in the design of the future generation of integrated observing systems. Preprint, 1st Symposium on Integrated Observation Systems, Long Beach, CA, Amer. Meteor. Soc., 45-47.

Lorenc, A. C., 1992: Iterative analysis using covariance functions and filters. Quart. J. Roy. Meteor. Soc., 118, 569-591.

McPherson, R., E. Kalnay, and S. Lord, 1997: The potential role of GPS/MET observations in operational numerical weather prediction. The Global Positioning System for the Geosciences, NRC Rep. 9254. National Academy Press [Available online at http://www.nap.edu/catalog/9254.html], 111-113.

Purser, R. J., 1987: The filtering of meteorological fields. J. Climate Appl. Meteor., 26, 1764-1769.

Ware, R., and Coauthors, 1996: GPS sounding of the atmosphere from low earth orbit: Preliminary results. Bull. Amer. Meteor. Soc., 77, 19-40.

Weckwerth, T. M., and D. B. Parsons, 2006: A review of convection initiation and motivation for IHOP_2002. Mon. Wea. Rev., 134, 5-22.

Weckwerth, T. M., J. W. Wilson, and R. M. Wakimoto, 1996: Thermodynamic variability within the convective boundary layer due to horizontal convective rolls. Mon. Wea. Rev., 124, 769-784.

Weckwerth, T. M., and Coauthors, 2004: An overview of the International $\mathrm{H}_{2} \mathrm{O}$ Project (IHOP_2002) and some preliminary highlights. Bull. Amer. Meteor. Soc., 85, 253-277.

Weckwerth, T. M., C. R. Pettet, F. Fabry, S. Park, M. A. LeMone, and J. W. Wilson, 2005: Radar refractivity retrieval: Validation and application to short-term forecasting. J. Appl. Meteor., 44, 285-277.

Xue, M., and W. J. Martin, 2006a: A high-resolution modeling study of the 24 May 2002 case during IHOP. Part II: Horizontal convective rolls and convective initiation. Mon. Wea. Rev., 134, 172-191.

Xue, M., and W. J. Martin, 2006b: A high-resolution modeling study of the 24 May 2002 case during IHOP. Part I: Numerical simulation and general evolution of the dryline and convection. Mon. Wea. Rev., 134, 149-171.

Xue, M., D.-H. Wang, J.-D. Gao, K. Brewster, and K. K. Droegemeier, 2003: The Advanced Regional Prediction System (ARPS), storm-scale numerical weather prediction and data assimilation. Meteor. Atmos. Phys., 82, 139-170. 